\title{
Insulin binding monitored by fluorescence correlation spectroscopy
}

\author{
Z-H.Zhong ${ }^{1}$, A.Pramanik ${ }^{2}$, K. Ekberg ${ }^{1}$, O.T.Jansson ${ }^{1}$, H.Jörnvall ${ }^{2}$, J. Wahren ${ }^{1}$, R. Rigler ${ }^{2}$ \\ ${ }^{1}$ Department of Surgical Sciences, Section of Clinical Physiology, Karolinska Hospital, Stockholm, Sweden \\ ${ }^{2}$ Department of Medical Biochemistry and Biophysics, Karolinska Institute, Stockholm, Sweden
}

\section{Abstract}

Aim/hypothesis. The characteristics of insulin binding to its receptors have been extensively studied by the radioligand binding assay. We used fluorescence correlation spectroscopy to determine the distribution of diffusion times and further novel data on the kinetics of insulin's binding to its receptor.

Methods. Cultured human renal tubular cells (HRTC) were incubated with tetramethyl rhodamine labelled insulin (Rh-Ins) for $60 \mathrm{~min}$. Fluorescence intensity fluctuations and autocorrelation functions for $\mathrm{Rh}$-Ins, free in the incubation medium and bound to the cell membrane, were studied at single-molecule detection sensitivity in a $0.2 \mathrm{fL}$ confocal volume.

Results. Measurements at the cell membrane revealed Rh-Ins binding with at least two diffusion components (diffusion times $\tau_{\mathrm{D} 1}=0.8 \mathrm{~ms}, \tau_{\mathrm{D} 2}=20 \mathrm{~ms}$ ) and corresponding weight fractions of $\mathrm{y}_{1}=0.43$ and $\mathrm{y}_{2}=0.42$. Specificity of the binding was shown by the dislocation of bound Rh-Ins when excess unlabelled insulin was added. Scatchard analysis showed a nonlinear plot, revealing two binding processes with different affinities $\left(\mathrm{K}_{\mathrm{ass}} \sim 2 \cdot 10^{10} \mathrm{M}^{-1}\right.$ and $\sim 1 \cdot 10^{9} \mathrm{M}^{-1}$, respectively).

Conclusion/interpretation. The fluorescence correlation spectroscopy results show two classes of binding sites with different affinities for insulin, or interactions between receptor sites consistent with negative cooperativity. This conclusion is in agreement with studies of insulin binding using radioligand binding assays. Because of its high sensitivity (single molecule detection), FCS, provides additional data allowing a more precise evaluation of the kinetics of ligand-receptor interactions at low expression levels in living cells. [Diabetologia (2001) 44: 1184-1188]

Keywords Insulin, fluorescence correlation spectroscopy, radioligand binding assay, cell membrane interaction, ligand receptor complex, tetramethyl rhodamine, human renal tubular cells, auto correlation function, scatchard analysis, diffusion times.
The development of methods for labelling hormones at high specific radioactivity allowed direct studies of hormone-receptor interactions without altering their biological activity. The radioligand binding assay has proved to be a powerful tool for evaluating receptor-ligand characteristics for a great number of

Received: 23 October 2000 and in revised form: 26 March 2001

Corresponding author: John Wahren, M. D., Section of Clinical Physiology, Karolinska Hospital, SE-17176 Stockholm, Sweden, E-mail: john.wahren@ks.se

Abbreviations: FCS, Fluorescence correlation spectroscopy; HRTC, human renal tubular cells; Rh-Ins, tetramethyl rhodamine labelled insulin. hormones. However, in specific situations, for example when the number of receptors for each unit cell surface area is small or when the half-life of the receptor-ligand complex is shorter than the duration of the washing steps required for separation of the bound and free hormone, observing specific binding can become difficult.

Few alternatives to the radioligand binding assay are available. However, fluorescence correlation spectroscopy (FCS) is now emerging as a biophysical technique with a high degree of specificity [1]. This technique records the thermodynamic fluctuations of single fluorescently labelled molecules after excitation by a focused laser beam $[2,3,4]$. In FCS, the fo- 
cus of the laser forms a small volume element $(0.2 \mathrm{fL})$ that allows the detection of movements of single molecules in biological systems $[5,6]$. Thus, the binding of a labelled ligand to its receptor on an intact cell can be detected [7]. The diffusion times for bound and unbound ligands and the average number of molecules in the volume of measurement can be calculated from the autocorrelation function of the fluctuations in fluorescence intensity. The FCS method has been used to show specific binding to cell membranes of epidermal growth factor, the neuropeptide galanin, and proinsulin C-peptide $[1,7,8]$. We applied the FCS technique to study insulin-receptor interactions.

\section{Materials and methods}

Cell culture. Human renal tubular cells were cultured from the outer cortex of renal tissue obtained from non-diabetic patients undergoing elective nephrectomy for renal cell carcinomas. The cells were cultured in RPMI 1640 (Life Technologies, Paisley, PA, USA) supplemented with $10 \%$ fetal calf serum, $2 \mathrm{mmol} / \mathrm{l}$ L-glutamine, $10 \mathrm{mmol} / \mathrm{l}$ HEPES, bensylpenicillin $(100 \mathrm{U} / \mathrm{ml})$ and streptomycin $(100 \mu \mathrm{g} / \mathrm{ml})$ and passaged at near confluence by trypsinization. Cells from the second and third passages were used for experiments. Tissue collection was undertaken with the informed consent of the subject and the approval of the institutional ethics committee.

Labelling of insulin. Human insulin (Novo Nordisk, Copenhagen, Denmark) was labelled with tetramethyl rhodamine (Rh-Ins) (absorption $555 \mathrm{~nm}$, emission $580 \mathrm{~nm}$ ), using a succinimidyl ester derivative (FluoReporter, F-6163 Molecular Probes Europe BV, Netherlands) at five to tenfold molar excess of the reagent. Rh-Ins was separated by passage through a NAP-5 column (Amersham Pharmacia Biotech, Uppsala, Sweden), and by reverse phase chromatography (Hewlett Packard 1090 HPLC) using a Kromasil C8 column $(4.6 \cdot 250 \mathrm{~mm}$; particle size $7 \mu \mathrm{m}$; pore size $10 \mathrm{~nm}$, Eka Nobel, Surte, Sweden), elution at $1 \mathrm{ml} / \mathrm{min}$ with a gradient of $20-40 \%$ acetonitrile containing $0.1 \%$ trifluoroacetic acid and detection at 555 and $220 \mathrm{~nm}$. Fractions containing Rh-Ins were pooled, adjusted to $\mathrm{pH} 8$ and lyophilized. The identities of the purified labelled peptide was confirmed by matrix assisted laser desorption ionization mass spectroscopy. Bioactivity of Rh-Ins was evaluated by incubation of L6 myoblasts with ${ }^{14} \mathrm{C}$-glucose and $\mathrm{Rh}$-Ins and insulin, respectively. Incorporation of labelled glucose into glycogen was almost identical for Rh-Ins and insulin.

Procedure. Binding studies with FCS were carried out [7]. Cells were cultured in eight-well Nunc chambers (Nalge Nunc Inc., Naperville Ill., USA) at $20^{\circ} \mathrm{C}$. Before the experiments, cells were washed five times with PBS (phosphate buffered saline) and incubated with binding buffer (20 mmol/1 HEPES, pH 7.4, $115 \mathrm{mmol} / 1 \mathrm{NaCl}, 24 \mathrm{mmol} / \mathrm{l} \mathrm{NaHCO}_{3}, 4.7 \mathrm{mmol} / \mathrm{l} \mathrm{KCl}$, $1.26 \mathrm{mmol} / 1 \mathrm{CaCl}_{2}, 1.2 \mathrm{mmol} / \mathrm{KH}_{2} \mathrm{PO}_{4}, 1.2 \mathrm{mmol} / 1 \mathrm{MgSO}_{4}$, $11.1 \mathrm{mmol} / \mathrm{l}$ glucose, and $5 \mathrm{mg} / \mathrm{ml}$ bovine serum albumin). Binding of insulin was measured after $60 \mathrm{~min}$ incubation in the presence of $0.1-5 \mathrm{nmol} / \mathrm{l} \mathrm{Rh}$-Ins. Specificity of insulin binding was demonstrated by competitive displacement of bound RhIns after $1-3 \mathrm{~h}$ incubation of $5 \mu \mathrm{mol} / \mathrm{l}$ non-labelled insulin added to the cell incubations (post-incubation). Specific binding was also shown by the inability of Rh-Ins to bind to cell surfaces that had been pre-treated with non-labelled insulin (pre-incubation).

Fluorescence correlation spectroscopy. FCS was done in a ConfoCor instrument (Carl Zeiss-Evotec, Jena, Germany) equipped with a Zeiss Neofluar 40X, N. A. 1.2 objective, a dichroic mirror (Omega 540 DRL PO2) and bandpass filters (Omega 565 DR 50 [7]). The Rh-Ins was excited with the $514.5 \mathrm{~nm}$ line of an Argon laser. Intensity fluctuations were detected by an avalanche photo diode (EG \& SPCM 200) and processed with a digital correlator (ALV 5000, ALV, Langen, Germany).

Evaluation of FCS data. Fluorescence intensity fluctuations $<\delta \mathrm{I}>$ around the mean fluorescence intensity $<\mathrm{I}>$ occurring in a volume element $(0.2 \mathrm{fL})$ with half axes $\omega=0.25 \mu \mathrm{m}$ and $\mathrm{z}=1.25 \mu \mathrm{m}$ were correlated [2-4] and the autocorrelation function, $\mathrm{G}(\tau)$, was evaluated by non-linear least square minimization [9]. $\mathrm{G}(\tau)$ for three dimensional diffusion of the unbound Rh-Ins in solution and two dimensional diffusion of bound Rh-Ins to membranes on the cell surface is given by:

$$
\begin{aligned}
& G(\tau)=1+\frac{\langle\delta I(t) \delta I(t+\tau)\rangle}{\langle I\rangle^{2}} \\
& =1+\frac{1}{N}\left[\left(1-\sum y_{i}\right)\left(\frac{1}{1+\frac{\tau}{\tau_{D}^{f}}}\right)\left(\frac{1}{1+\left(\frac{\omega}{z}\right)^{2} \frac{\tau}{\tau_{D}^{s}}}\right)^{\frac{1}{2}}+\sum y_{i}\left(\frac{1}{1+\frac{\tau}{\tau_{D}^{s}}}\right)\right]
\end{aligned}
$$

where diffusion time $\tau_{D}$ and diffusion coefficient $D$ are related as

$\tau_{\mathrm{D}}=\frac{\omega^{2}}{4 D}$

$y_{i}$ is the fraction of membrane bound Rh-Ins diffusing with diffusion time $\tau_{D}^{b i}$ and $\left(1-\sum y_{i}\right)$ is the fraction of unbound Rh-Ins diffusion with diffusion time $\tau_{D}^{f}$.

The amplitude of the $G(\tau)$ is proportional to the inverse number of fluorescent ligand molecules $(N)$ in the volume element. The relative amount of cell surface bound insulin ligand $\left(\Sigma y_{i}\right)$ increases in keeping with increasing concentration of $\mathrm{Rh}$-Ins. The Scatchard representation of the mass action law is obtained from:

$\frac{\sum \mathrm{y}_{\mathrm{i}}}{\left(1-\sum \mathrm{y}_{\mathrm{i}}\right) \mathrm{R}_{0}}=\sum K_{\mathrm{i}}\left(\mathrm{n}_{\mathrm{i}}-\frac{\mathrm{y}_{\mathrm{i}} \mathrm{L}_{0}}{\mathrm{R}_{\mathrm{o}}}\right)$

where $K_{i}$ and $n_{i}$ are the association constant and the number of ligand binding sites for each receptor molecules, respectively, $L_{o}$ is the total ligand concentration. The total receptor concentration, $R_{o}$, could not be determined independently but was calculated from the maximum number of bound ligand molecules, $L_{b}$, at saturation, assuming one binding site for one receptor molecule $(n=1)$. A distribution function $P\left(\tau_{D i}\right)$, describing the fraction of species $y_{i}$, was calculated from the correlation function $G(\tau)$ :

$G(\tau)=1+\frac{1}{N} \int_{i} P\left(\tau_{D_{i}}\right) \frac{1}{1+\frac{\tau}{\tau_{D_{i}}}}\left(\frac{1}{1+\left(\frac{\omega}{z}\right)^{2} \frac{\tau}{\tau_{D_{i}}}}\right)^{\frac{1}{2}} d \tau_{D_{i}}$

with $P\left(\tau_{D i}\right)=\Sigma y_{i}$ dy. Further evaluation of $P\left(\tau_{D i}\right)$ was performed by the CONTIN programme using restrained regularization [10].

\section{Results}

Fluorescence intensity fluctuations and autocorrelation functions for free Rh-Ins in solution and bound 

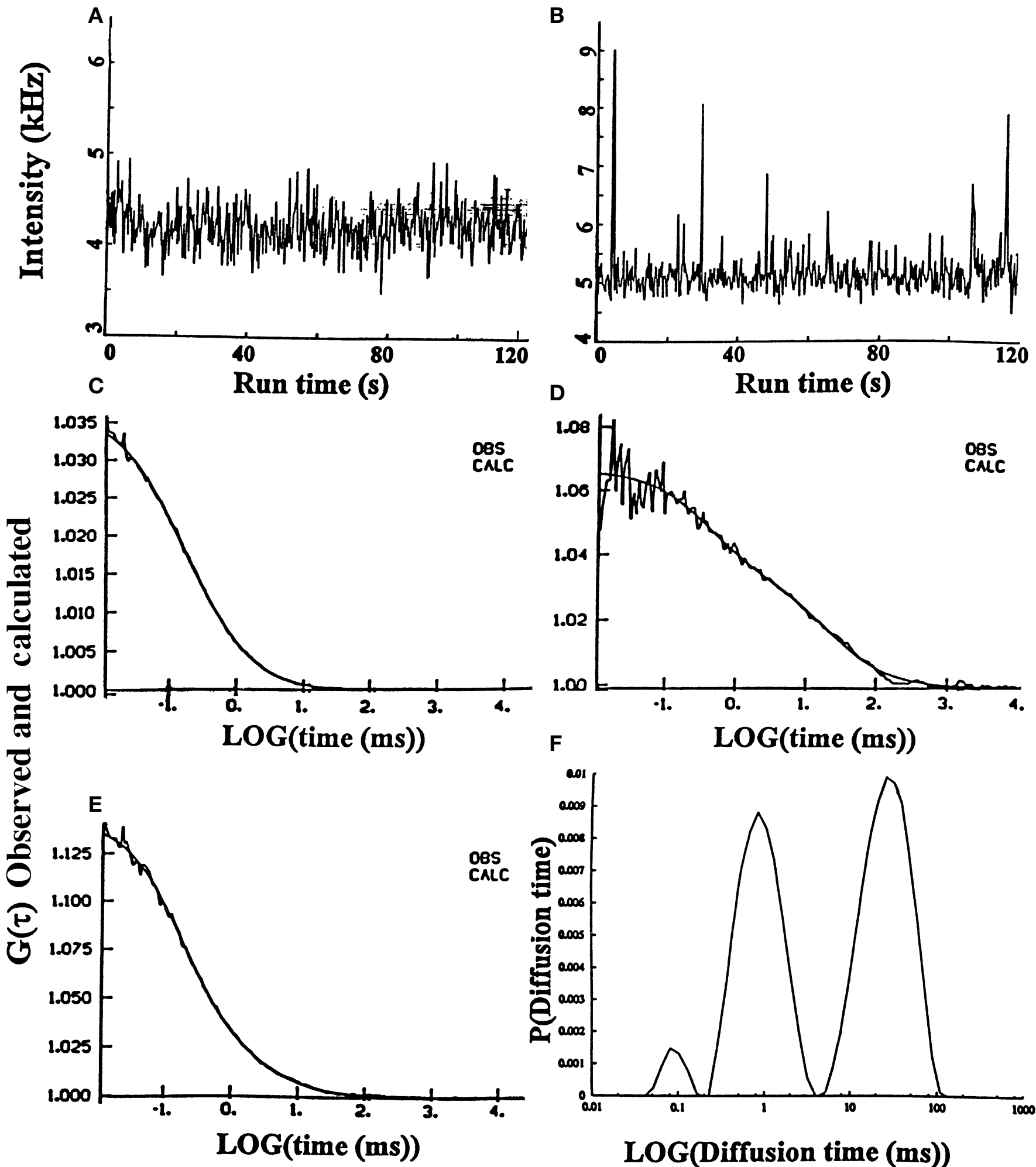

Fig. 1. Insulin binding and displacement to the membranes of cultured renal tubular cells. Fluorescence intensity fluctuations (A) and autocorrelation function $(\mathbf{C})$ for Rh-Ins (5 $\mathrm{nmol} / \mathrm{l})$ free in solution, $\tau_{D}=0.11 \mathrm{~ms}$. Fluorescence intensity fluctuations (B) and autocorrelation function (D) for Rh-Ins bound to membranes on the cell surfaces. Diffusion times $(\tau)$ and corresponding fractions $(y): \tau_{D 1}=0.8 \mathrm{~ms}, y_{1}=0.43$;

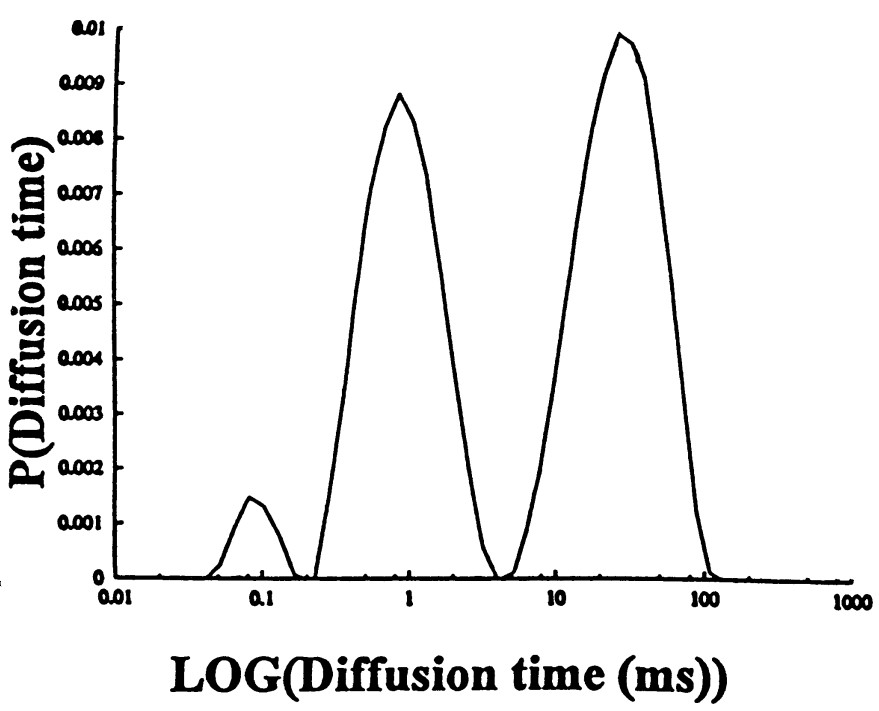

$\tau_{D 2}=20 \mathrm{~ms}, y_{2}=0.42 ; \tau_{D 3}=0.11 \mathrm{~ms}, y_{3}=1-\left(y_{1}+y_{2}\right)=0.15$. Autocorrelation function following displacement of membrane bound $\mathrm{Rh}$-Ins by postincubation of a thousand fold molar excess of non-labelled insulin: $\tau_{D 1}=1.3 \mathrm{~ms}, y_{1}=0.09$; $\tau_{D 3}=0.11 \mathrm{~ms}, y_{3}=0.91(\mathbf{E})$. CONTIN distributions of diffusion times $\mathrm{P}\left(\tau_{D i}\right)$ of Rh-Ins bound to the cell membranes $(\mathbf{F})$ 


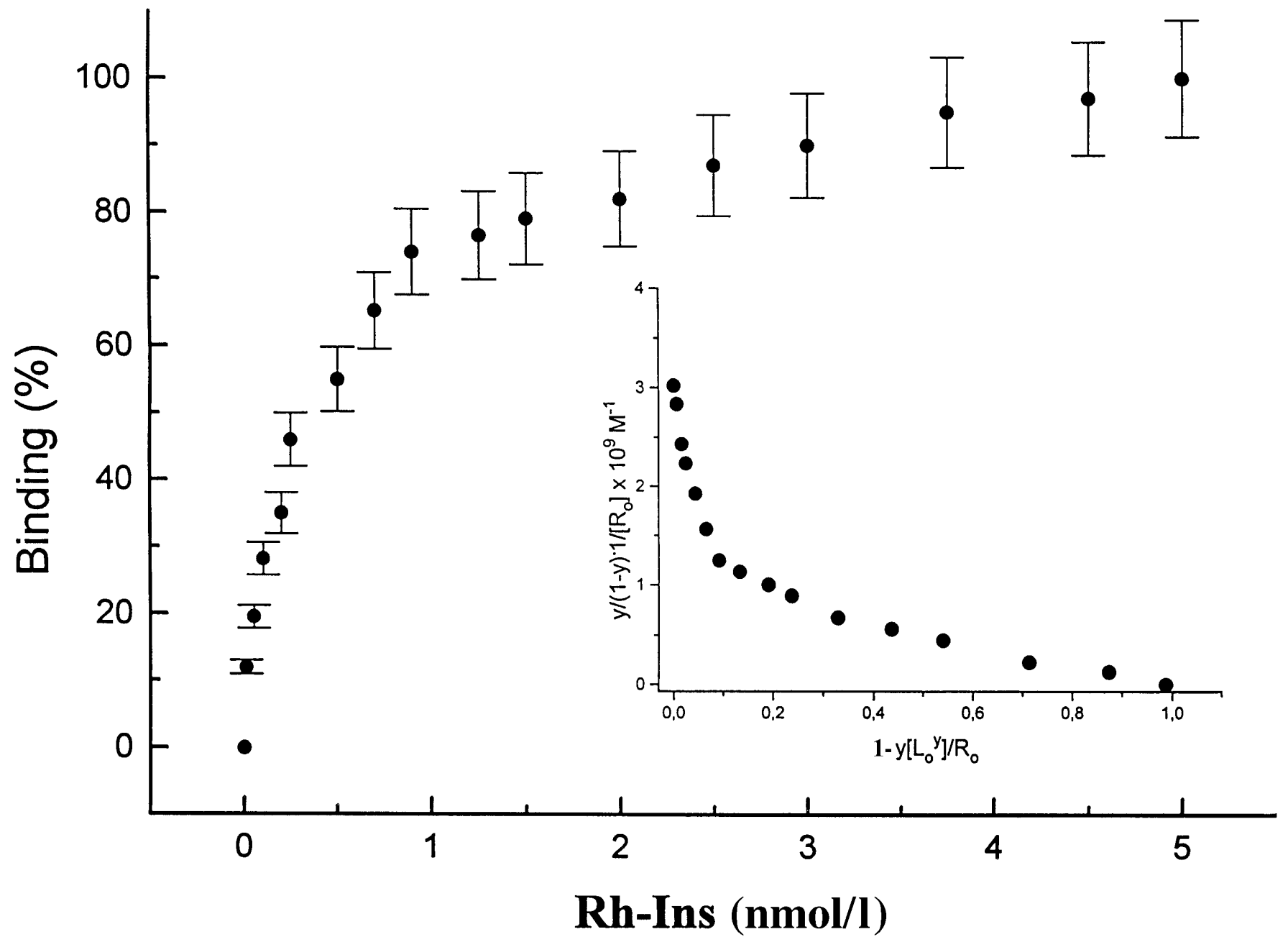

Fig. 2. Insulin binding curve. Binding of Rh-labelled insulin to the cell membranes of renal tubular cells. Fractional saturation of the membrane bound Rh-Ins ( $y$ in equation 1 ) as a function of the ligand concentration (L) in the binding medium. Each data point represents the mean of six measurements. Means $\pm \mathrm{SE}$ are indicated. The Scatchard plot (insert) indicates the presence of two binding process with the association constants $\sim 1 \cdot 10^{9} \mathrm{M}^{-1}$ and $\sim 2 \cdot 10^{10} \mathbf{M}^{-1}$, respectively

to cell membranes are illustrated in Figure 1. Free $\mathrm{Rh}$-Ins in the buffer medium superficial to the cells showed characteristic Brownian motion and fluorescence fluctuations (Fig. 1A), and an average diffusion time $(\tau)$ of $0.11 \mathrm{~ms}$ (Fig. 1C). When the volume element was positioned at the level of the cell membrane, an increase and a broadening of the fluorescence fluctuations were observed (Fig. 1B). Autocorrelation analysis of the intensity fluctuations then showed a diffusion process of the cell-bound $\mathrm{Rh}$-Ins, with at least two components characterized by diffusion times of $\tau_{D 1}=0.8 \mathrm{~ms}$ and $\tau_{D 2}=20 \mathrm{~ms}$, respectively, and corresponding weight fractions of $y_{1}=0.43$ and $y_{2}=0.42$ (Fig. 1D). The volume element extends into the space above the cell membrane and a small component of unbound Rh-Ins $\left(\tau_{D 3}=0.11, y_{3}=0.15\right)$ was also recorded. Addition of 1000 -fold molar excess of unlabelled insulin resulted in an almost complete displacement of the bound Rh-Ins (Fig.1E). Likewise, preincubation with excess unlabelled insulin resulted in failure of Rh-Ins to bind (data not shown). A model-independent analysis of the diffusion processes of bound $\mathrm{Rh}$-Ins is presented in Fig. 1F, showing the distribution of the three diffusion times $\tau_{D 1}, \tau_{D 2}$ and $\tau_{D 3}$.

An insulin binding isotherm was obtained at increasing concentrations of Rh-Ins (Fig. 2). From the Scatchard representation (Fig. 2, insert) of the binding isotherm, a binding process extrapolating to one $\mathrm{Rh}$-Ins molecule for each receptor with an association constant of about $1 \cdot 10^{9} \mathrm{M}^{-1}$ was observed. In addition, evidence for the existence of a second binding process with higher affinity $\left(K_{\text {ass }}=\sim 2 \cdot 10^{10} \mathrm{M}^{-1}\right)$ and a ligand-receptor complex involving at least two receptor molecules for each insulin molecule $(n<1)$ was also observed. The higher affinity part of the Scatchard representation corresponds to the first part of the binding isotherm, where the ligand is present at low concentrations. The second part of the binding isotherm, obtained at higher Rh-Ins concen- 
trations, corresponds to the low affinity interaction of the Scatchard plot.

\section{Discussion}

In FCS measurements, fluorescence intensity fluctuations are recorded only from those molecules that diffuse through the minute confocal laser volume element $(0.2 \mathrm{fl})$. The time required for the passage of molecules through the volume element is determined by the diffusion coefficient, which is related to the molecular size. Thus, diffusion times obtained from the analysis of fluorescence intensity fluctuations with autocorrelation functions allow differentiation between rapidly and slowly diffusing molecules, which serves as an analogy to unbound and bound states of ligand molecules. Because the two diffusion times, $\tau_{D 1}=0.8 \mathrm{~ms}$ and $\tau_{D 2}=20 \mathrm{~ms}$ observed for RhIns, are much longer than the $\tau_{D 3}=0.11 \mathrm{~ms}$ of unbound Rh-Ins (Fig. 1D and C), they correspond to the ligand-receptor complexes. These results show the specific binding of insulin to its membrane-bound receptors; the specificity of the binding is confirmed by the consistent displacement of bound Rh-Ins following addition of 1000-fold molar excess of unlabelled insulin.

The Scatchard plot (Fig. 2, insert) illustrates two binding processes with a high affinity $K_{\text {ass }}$ of about $2 \cdot 10^{10} \mathrm{M}^{-1}$ and a low affinity $K_{\text {ass }}$ of about $1 \cdot 10^{9}$ $\mathrm{M}^{-1}$. The documentation of two separate binding complexes moving with different diffusion times (Fig.1F), reflects the complex nature of insulin's binding to its receptor. The results are compatible either with two binding-sites having low and high affinity, respectively, for insulin or the presence of negative cooperativity, i.e. binding of one ligand results in reduction of receptor affinity for subsequent ligand molecules $[11,12]$. Because the diffusion times of the ligand molecule and the ligand-receptor complexes are used in FCS as characteristic parameters for the analysis of binding, the FCS technique provides information that cannot be obtained with the conventional radioligand binding assay. The distribution of diffusion times for the ligand-receptor complexes allows the aggregation kinetics of individual ligand-receptor complexes to be studied, thereby providing additional information on the signal transduction process. Irrespective of the final evaluation of the insulin binding kinetics, it is clear from the present results that the FCS technique is highly sensitive and appropriate for studies of binding of insulin and hormones in general to cell membranes.

\section{References}

1. Rigler R (1995) Fluorescence correlations, single molecule detection and large number screening. Applications in biotechnology. J Biotechnol 41: 177-186

2. Magde D, Elson EL, Webb WW (1972) Thermodynamic fluctuations in a reacting system. Measurement by fluorescence correlation spectroscopy. Phys Rev Lett 29: 705-711

3. Ehrenberg M, Rigler R (1974) Rotational Brownian motion and fluorescence intensity fluctuation. Chem Phys 4: 390-401

4. Elson EL, Magde D (1974) Fluorescence correlation spectroscopy. I. Conceptual basis and theory. Biopolymers 13: $1-27$

5. Eigen M, Rigler R (1994) Sorting single molecules: application to diagnostics and evolutionary biotechnology. Proc Natl Acad Sci USA 91: 5740-5747

6. Maiti S, Haupts U, Webb W (1997) Fluorescence correlation spectroscopy: diagnostics for sparse molecules. Proc Natl Acad Sci USA 94: 11753-11757

7. Rigler R, Pramanik A, Jonasson P et al. (1999) Specific binding of proinsulin C-peptide to human cell membranes. Proc Natl Acad Sci USA 96: 13318-13323

8. Pramanik A, Juréus A, Langel Ü, Bartfai T, Rigler R (1999) Galanin receptor binding in the membranes of cultured cells measured by fluorescence correlation spectroscopy. Biomed Chromatogr 13: 119-120

9. Marquard DW (1963) An algorithm for least-squares estimation of nonlinear parameters. J Soc Indust Appl Math 11: 431-441

10. Provencher SW (1982) Contin: a general purpose constrained regulation program for inverting noisy linear algebraic and integral equations. Comput Phys Commun 27: 213-227

11. DeMeyts P, Roth J, Neville DM Jr, Gavin Jr 3rd, Lesniak MA (1973) Insulin interactions with its receptor: experimental evidence for negative cooperativity. Biochem Biophys Res Commun 55: 154-161

12. DeMeyts P (1994) The structural basis of insulin and insulin-like growth factor-1 receptor binding and negative cooperativity, and its relevance to mitogenic versus metabolic signalling. Diabetologia 37 [Suppl 2]: 135-148 